Research paper

\title{
Coherent structures in oscillatory flows within the laminar-to-turbulent transition regime for smooth and rough walls
}

\author{
ANNA MUJAL-COLILLES, Post Doctoral Researcher, Department of Civil and Environmental Engineering, Marine Engineering \\ Laboratory, UPC-Barcelona Tech, Barcelona, Spain \\ Email: anna.mujal@upc.edu (authorfor correspondence)
}

KENNETH T. CHRISTENSEN, Professor Colleg of Enginering Chair in Fluid

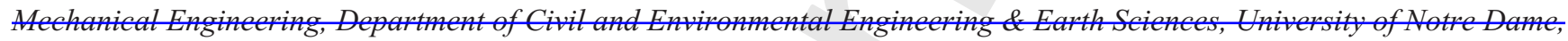
USA

Email: christensen.33@nd.edu

ALLEN BATEMAN (IAHR Member), Professor, Department of Hydraulic, Marine and Environmental Engineering, Sediment Transport Research Group (GITS). Barcelona Tech, Barcelona, Spain

Email: allen.bateman@upc.edu

MARCELO H. GARCIA (IAHR Member), Professor and Chair in Civil Engineering, Department of Civil and Environmental Engineering, University of Illinois, Urbana-Champaign, USA

Email:m.h.garcia@uiuc.edu

\begin{abstract}
We investigate coherent structures present in oscillatory boundary layers over smooth and rough beds for Reynolds numbers between $10^{3}$ and $10^{4}$, in the transition to turbulence regime. A two-camera 2D-particle image velocimetry (PIV) system was used to visualize coherent structures in an oscillatory-flow tunnel. The obtained results show that smooth-bed flow is populated by vortex-tube structures that are formed due to the KelvinHelmholtz instability. Three types of coherent structures are observed in the rough-wall experiments: vortices randomly distributed in space; turbulent bursts - spatial structures without a clear shape compared to vortices but with a longer life than the former; and shear layers of vortices originating due to flow separation from some of the grains on the bed. The reported study contributes to the description of coherent structures in oscillatory flows that are captured with the PIV technique, particularly a new structure in flows over rough beds in the transition to turbulence regime.
\end{abstract}

Keywords: Boundary layer turbulence; coherent structures; oscillatory flows; particle image velocimetry (PIV); transition to turbulence

\section{Introduction}

Unsteady flows are of great importance owing to their prevalence in nature and industry. Due to the difficulties in studying unsteady flows, canonical cases have been used to characterize, for example, acceleration and deceleration phases. Oscillatory flows are of particular interest because of their relevance in the study of biological processes such as human breathing cycles, nutrient cycling in plants, and also the physical processes involving sediment transport under wave action. These flows have additionally received a great deal of attention due to their simple driving mechanisms.

Laminar oscillatory flows were first studied in the context of the Stokes boundary layer with the theoretical foundations developed by Lamb (1932) and Batchelor (1967). Experimental studies began with the work by Li (1954) who first attempted to parameterize the relevant flow regimes. Kamphuis (1975) focused his experiments on the friction factor effects under oscillatory flows where he developed a Moody-type diagram and a corresponding flow regime distribution chart. More recently,

Received 22 December 2014; accepted 3 April 2016/Currently open for discussion. 
Pedocchi and Garcia (2009) proposed a methodology for estimating the friction coefficient in oscillatory flows in the transition from hydraulically smooth to fully rough flow conditions. Several authors have carried out many more experiments under a wide range of Reynolds numbers $\left(\mathrm{R}=A U_{0 m} / v\right.$, where $A$ is the amplitude of oscillation and $U_{0 m}$ is the maximum free-stream velocity) and different wall conditions (e.g. Hino, Nakayama, Kashiwayanagi, \& Hara, 1983; Jensen, Sumer, \& Fredsoe, 1989; Jonsson, 1980; Sleath, 1987; Sumer, Jensen, \& Fredsoe, 1987). Many of these previous studies focused on defining oscillatory flows using an approach analogous to that typically used for steady flow scenarios. For instance, Jonsson (1980) was the first to propose a time-dependent logarithmic law that is valid for most of the flow phases in the turbulent regime. This formulation was improved by Sleath (1987) with a larger number of experiments, and further by Jensen et al. (1989).

Several studies have specifically focused on the existence of turbulence in oscillatory smooth- and rough-wall flows over a range of $\mathrm{R}$ (e.g. Blondeaux, Scandura, \& Vittori, 2004; Hino et al., 1983; Jensen et al., 1989; Sleath, 1987). In general, it is understood that turbulence appears during the latter stages of the acceleration phases and is maintained during the deceleration half cycle. At the early stages of the acceleration phase, flow can revert back to the laminar regime depending on $\mathrm{R}$.

Coherent structures are a key feature of any transitional or fully turbulent flow. They drive transport and mixing processes as well as induce significant drag at solid surfaces. In the context of oscillatory flows, a recent study by Carstensen, Sumer, and Fredsøe (2010) highlights the existence of such coherent motions in the transitional regime. They described two different structures depending on $\mathrm{R}$. The first type, termed vortex tubes (for $\mathrm{R}<3 \times 10^{5}$ ), appears as $2 \mathrm{D}$ spanwise-rotating tubes emerging at the end of the deceleration phase, and disappearing during the later stages of the acceleration phase. Carstensen et al. (2010) hypothesized that these vortex tubes originated due to the Kelvin-Helmholtz instability that develops in the shear layer at motion phases prior to the flow reversal. The second observed structure, termed turbulent spots $\left(\mathrm{R}>1.5 \times 10^{5}\right)$, is highly three-dimensional (3D) in shape and appeared randomly in space in a manner similar to that in the classical transitional boundary-layer flows (i.e. with a unidirectional free-stream). Mazzuoli, Vittori, and Blondeaux (2011) utilized numerical simulations to study the origin of the latter structures, following the scheme used previously by Vittori and Verzicco (1998) and Costamagna, Vittori, and Blondeaux (2003).

For oscillatory rough-wall flow, Jensen et al. (1989) described the revealed structures as jets and bursts, with the former being specifically associated with individual roughness elements and occurring at specific phases (Sleath, 1987). Fornarelli and Vittori (2009) performed numerical simulations over a rough wall formed by a periodic pattern of hemispheres following the experiments of Keiller and Sleath (1976). Both studies described the formation of near-wall shear layers during the acceleration phases, similar to the jets described previously by Sleath (1987).

The present study utilized planar particle-image velocimetry (PIV) to capture the coherent flow structures present in oscillatory flows under the laminar to turbulent transition regime over smooth and rough beds in the streamwise-wall-normal plane. The formation, evolution and life of the observed structures were identified within the PIV velocity fields acquired within the transition-to-turbulent regime using periodic oscillatory conditions. The smooth-wall results reveal structures consistent with the vortex tubes described by Carstensen et al. (2010). However, as the experiments presented herein employ steady oscillations with a fixed period, phase-averaging methods are exploited to describe the coherent structures at specific phases of the oscillatory flow.

\section{Experimental set-up}

196

Experiments were conducted in the Ven Te Chow Hydrosystems Laboratory at the University of Illinois at Urbana-Champaign in a U-shaped oscillatory flow water tunnel (Admiraal, García, \& Musalem, 2004). Figure 1 presents a sketch of the flume which has a working section approximately $0.25 \mathrm{~m}$ high, $0.20 \mathrm{~m}$ wide, and $4 \mathrm{~m}$ long. The surface and side walls are made of smooth transparent Perspex which facilitates optical access to the flow from the sides and above, while the bed upon which the

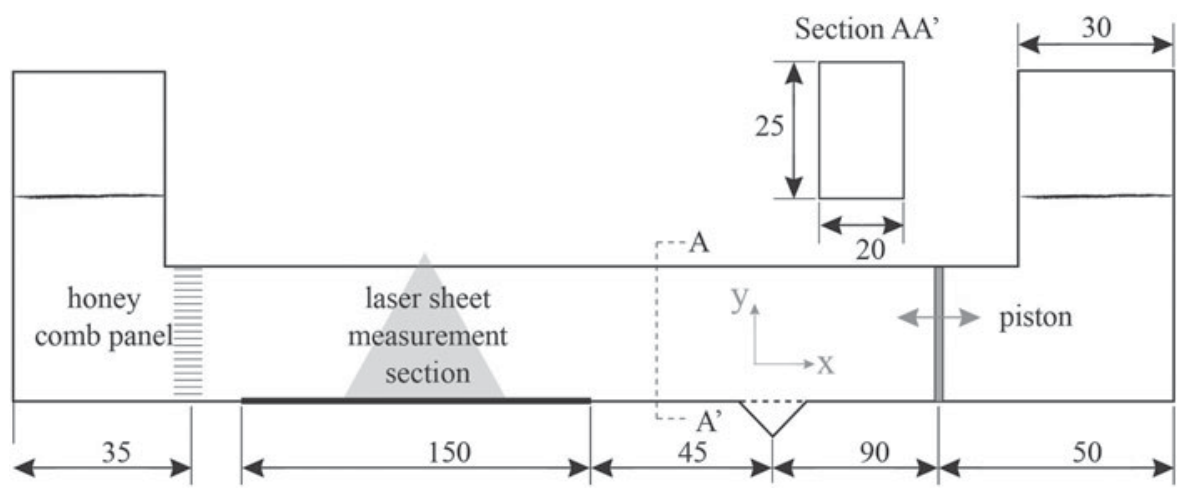


smooth-wall oscillatory boundary layers developed is composed of a bare polyvinyl chloride (PVC) plate. Gravel was glued to the surface of the PVC plate for the rough bed scenarios.

The oscillatory flow was driven by an Exlar electrical actuQ10 ator powering a piston located at the downstream end of the 226 flume. The actuator was controlled by a computer through Q11 a National Instruments card and a custom program devised with LabView which generated a repeatable sinusoidal motion.

Q12 Cycles were recorded continuously without stopping the actuator, but not consecutively and so they must be considered 231 independent experimental runs. The sinusoidal motion was set using an amplitude control (accuracy to within $0.1 \mathrm{~mm}$ ) and a period control with $1 \mu \mathrm{s}$ accuracy. A honeycomb structure was placed across the entire cross-section at the upstream inlet of the flume to suppress flow disturbances generated at the junction of the flume and the equilibrium chimney. The laser sheet measurement section was positioned far from the transition between Perspex and PVC to ensure uniform flow characteristics at each phase (Admiraal et al., 2004).

Table 1 describes the flow conditions measured for the three bed scenarios: a smooth bed, a small-grain rough bed $\left(D_{50}=1.5 \mathrm{~mm}, \sigma_{D}=1.3 \mathrm{~mm}\right)$ and a large-grain rough bed (with $D_{50}=7.0 \mathrm{~mm}, \sigma_{D}=1.2 \mathrm{~mm}$ ). In Table $1, T$ is the period of the oscillation and $\delta$ is the measured height of the boundary layer (Mujal-Colilles, Mier, Christensen, Bateman, \& Garcia, 2013). In terms of nomenclature, scenarios refer to the three different wall conditions (smooth, small roughness and large roughness), while cases refer to the four $\mathrm{R}$ values used in each scenario. The term test refers to a case-scenario combination.

Figure 2 categorizes all flow regimes studied herein using the classification of Kamphuis (1975). The smooth-wall scenarios fall close to the line dividing the laminar and turbulent regimes. Although the limits between these regimes are not perfectly defined, measured velocity profiles show how Tests 3 and 4 may be considered in the laminar regime whereas Tests 1 and 2 appear to be in the laminar-to-turbulent transition zone. However, in real terms all smooth-wall scenarios shall be considered to be in the laminar-to-turbulent regime, as reported by

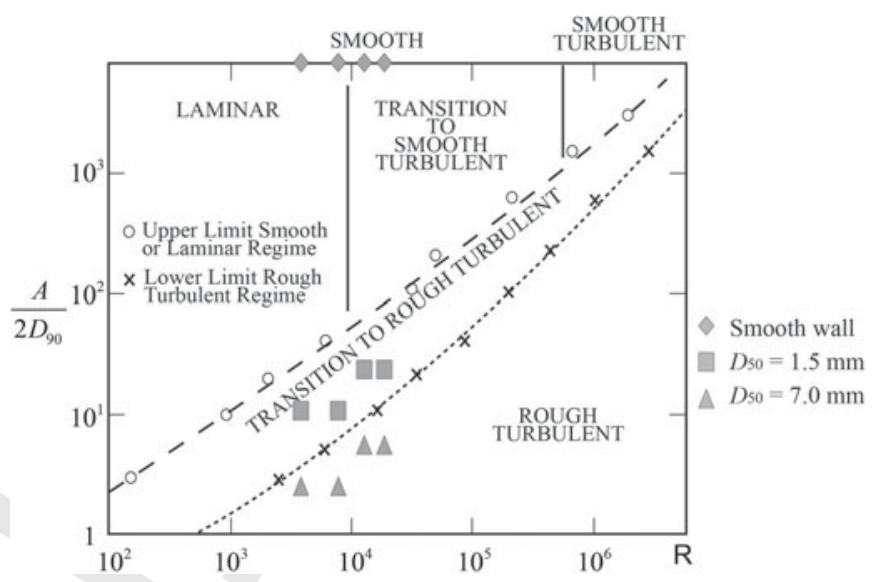

276

281

286

Figure 2 Diagram defining the flow regime (adopted from Kamphuis, 1975). Smooth wall scenarios are close to the $R$ values separating the laminar regime from the transition regime. Smaller bed roughness scenarios are within the transition to rough turbulent and finally larger bed roughness scenarios fall close to the border between the transition regime and the rough turbulent

Mujal-Colilles et al. (2013). Scenarios tested with the smaller roughness clearly sit within the smooth-to-rough transition zone. Finally, the larger roughness scenarios fall within the rough turbulent regime but in a region very close to the transition zone, suggesting that most of the oscillatory phases in our study are likely within the transition zone with only a few extending into the rough turbulent regime. A detailed discussion of these flow regime classifications for the present experiments can be found in Mujal-Colilles et al. (2013).

Instantaneous velocity fields were measured at the centrespan of the tunnel using a planar PIV system consisting of a Gemini PIV Nd:YAG pulse laser (New Wave Research) and two 4 MPx, 12-bit frame-straddle charge-coupled device (CCD) cameras (TSI Inc.). The two cameras were set on opposite Q14 sides of the flume and offset in the streamwise direction so that the individual fields of view overlapped by $6.3 \mathrm{~mm}$ (Fig. 3) to facilitate a wide composite streamwise field of view. Each camera was mounted to an aluminium frame and set $55 \mathrm{~cm}$ from

Table 1 Test conditions for the smooth- and rough-wall flow cases. Roughness parameter $k_{s}$, is used as $2 D_{50}$

\begin{tabular}{|c|c|c|c|c|c|c|c|c|}
\hline & Test no. & $T(\mathrm{~s})$ & $A(\mathrm{~mm})$ & $U_{0 m}\left(\mathrm{~mm} \mathrm{~s}^{-1}\right)$ & $\mathrm{R}\left(10^{4}\right)$ & $\Delta(\omega t)$ & $\delta(\mathrm{mm})$ & $A / k_{s}$ \\
\hline \multirow[t]{4}{*}{ Smooth } & 1 & 3.3 & 100 & 188 & 1.86 & $15^{\circ}$ & 2.2 & - \\
\hline & 2 & 5 & 100 & 125 & 1.27 & $10^{\circ}$ & 2.8 & - \\
\hline & 3 & 1.7 & 45.5 & 171 & 0.77 & $30^{\circ}$ & 1.6 & - \\
\hline & 4 & 3.3 & 45.5 & 86 & 0.38 & $15^{\circ}$ & 2.2 & - \\
\hline \multirow{4}{*}{$\operatorname{Rough}\left(D_{50}=1.5 \mathrm{~mm}\right)$} & 5 & 3.3 & 100 & 188 & 1.86 & $15^{\circ}$ & 3.6 & 33 \\
\hline & 6 & 5 & 100 & 125 & 1.27 & $10^{\circ}$ & 3.0 & 33 \\
\hline & 7 & 1.7 & 45.5 & 171 & 0.77 & $30^{\circ}$ & 2.0 & 15 \\
\hline & 8 & 3.3 & 45.5 & 86 & 0.38 & $15^{\circ}$ & 1.8 & 15 \\
\hline \multirow[t]{4}{*}{$\operatorname{Rough}\left(D_{50}=7.0 \mathrm{~mm}\right)$} & 9 & 3.3 & 100 & 188 & 1.86 & $15^{\circ}$ & 8.0 & 7 \\
\hline & 10 & 5 & 100 & 125 & 1.27 & $10^{\circ}$ & 8.6 & 7 \\
\hline & 11 & 1.7 & 45.5 & 171 & 0.77 & $30^{\circ}$ & 2.0 & 3 \\
\hline & 12 & 3.3 & 45.5 & 86 & 0.38 & $15^{\circ}$ & 2.8 & 3 \\
\hline
\end{tabular}




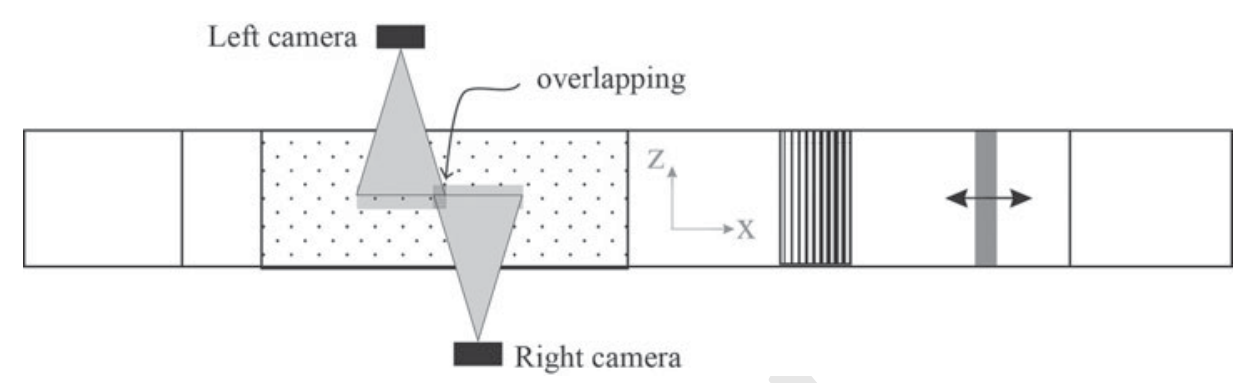

Right camera

Figure 3 Top view of the experimental set-up: camera locations. Two 4 Mpx CCD cameras were used

the measurement plane defined by the laser light sheet. The combined field of view was $4 \mathrm{~cm}$ high and $8 \mathrm{~cm}$ long [wallnormal $(y)$ by streamwise $(x)$ ]. The light sheet had a thickness of $0.5 \mathrm{~mm}$ at the channel bottom, and was optimized with a combination of cylindrical and spherical lenses with focal lengths of $15 \mathrm{~mm}$ and $500 \mathrm{~mm}$, respectively. The tracer particles used for the PIV measurements were Sphericel ${ }^{\circledR}$ hollow glass spheres of $1 \mu \mathrm{m}$ mean diameter and a density of $1.1{\mathrm{~g}-\mathrm{cm}^{-3}}^{\text {(Potter }}$ Q15. Industries Inc.).

Table 1 summarizes all test conditions analysed for the smooth and rough scenarios. The velocity $U_{0 m}$ represents the amplitude of the free stream velocity defined as:

$$
U_{0}(\omega t)=-U_{0 m} \cos (\omega t)
$$

including a negative value at $t=0$, due to the free-stream velocity initial motion from right to left in the field of view. Here, $\omega=2 \pi / T$ represents the angular frequency of the oscillation. The results obtained using the boundary layer height, $\delta$, following a definition offered by Sumer et al. (1987), are shown in Table 1.

Measurements were made at a sampling rate of $7.25 \mathrm{~Hz}$, which was the maximum acquisition frequency allowed by the PIV system. Thus, the initial periods of oscillation considered $(T=5,3$, and $2 \mathrm{~s})$ were adjusted proportionally to the sampling rate to exactly fit the number of phases recorded in a cycle. Therefore, each experiment has a number of recorded image pairs proportional to 12 ; this represents an increment in phase between samples of $10^{\circ}$ when $T=5.007 \mathrm{~s}, 15^{\circ}$ when $T=3.338 \mathrm{~s}$ and $30^{\circ}$ when $T=1.669 \mathrm{~s}$. Sixty cycles were recorded for each scenario, a number slightly larger than the proposed by Sleath (1987) for convergence of mean statistics.

Analysis of the PIV image pairs was accomplished using the Insight 8 software (TSI Inc.). The final interrogation window size for all experimental runs was $16 \times 16 \mathrm{px}^{2}$, which was obtained by applying a recursive interrogation approach with an original interrogation window size of $64 \times 64 \mathrm{px}^{2}$. Nyquist's criterion was satisfied in all interrogation steps, meaning that the final interrogation area of $16 \times 16 \mathrm{px}^{2}$ overlapped by $50 \%$ yielded a vector grid spacing of $0.4 \mathrm{~mm} \times 0.4 \mathrm{~mm}$ in the final velocity fields. A local validation was performed between the recursive passes in order to eliminate erroneous vectors. Final validation was performed using three local median validations of different sizes, and two replacement steps for initially eliminated vectors, the last of which was performed using a smoothing window size of $3 \times 3$ grid points. The interrogation process was conducted separately for each camera image followed by a final merging of frames at the vector field level. The overlap between the camera fields of view was obtained with a simple matching code meant to minimize differences between the right and the left vector fields in the overlapping region. The final merging process consisted of a smooth transition between the two fields, thus avoiding any transition region in the middle of the composite field of view.

\section{Results}

Phase averaging from the 60 cycles acquired, as suggested by (Sleath, 1987), is used to explore phase-dependent flow behaviour for the various scenarios considered herein. In this context, the velocity fields can be analysed as a function of phase as:

$$
\begin{aligned}
U_{0}(\omega t) & =-U_{0 m} \cos (\omega t) \\
U^{i}(x, y, \omega t) & =-U_{m}^{i}(x, y) \cos (\omega t) \\
U(x, y, \omega t) & =\frac{1}{N} \sum_{i} U^{i}(x, y, \omega t) \\
U(x, y, \omega t) & =-U_{m}(x, y) \cos (\omega t)
\end{aligned}
$$

where the superscript $i$ denotes the cycle, the subscript $m$ is the maximum value of $U$, and $\omega$ is the frequency of the oscillation. Turbulence quantities were first computed for each fluctuation field and then averaged over the 60 cycles as shown below for the case of the Reynolds stress:

$$
\begin{aligned}
\overline{u^{\prime} v^{\prime}}(x, y, \omega t)= & \frac{1}{N} \sum_{i}\left[\left(U^{i}(x, y, \omega t)-U(x, y, \omega t)\right)\right. \\
& \left.\times\left(V^{i}(x, y, \omega t)-V(x, y, \omega t)\right)\right]
\end{aligned}
$$

The overbar indicates phase averaged variables and will only be used in this paper for velocity fluctuations (i.e. turbulence intensities). The rest of the variables are assumed to be phaseaveraged and overbar is omitted throughout the document.

The variable used herein to visualize the coherent structures within the planar PIV velocity fields is the swirling strength, $\lambda_{c i}^{2}$ (Zhou, Adrian, Balachandar, \& Kendall, 1999), adapted by 
441 Adrian, Christensen, and Liu (2000) for vortex core identification in planar velocity fields. It is frame-independent and only identifies regions of local flow rotation in contrast to vorticity, which can be tainted by regions of intense shear as are often present in the near-wall regions of the wall-bounded flows under study (Adrian et al., 2000; Wu \& Christensen, 2006).

\subsection{Smooth wall}

Carstensen et al. (2010) defined two different types of coherent structures for oscillatory flows with increasing velocity amplitude over smooth-wall beds: vortex tubes $\left(7 \times 10^{4}<\mathrm{R}<3 \times 10^{5}\right)$ and turbulent spots $\left(\mathrm{R}>1.5 \times 10^{5}\right)$. The ranges of $R$ studied herein are below the limits described by Carstensen et al. (2010) for both structures (Table 1). Nevertheless, streamwise-averaged turbulence intensities plotted in Fig. 4 reveal the existence of significant velocity fluctuations in these tests which implies the possible existence of underlying coherent structures. As expected, higher levels of turbulence intensities are present at later phases of the acceleration half cycle $\left(90^{\circ}<\omega t<180^{\circ}\right)$, which are maintained during the deceleration cycle $\left(0^{\circ}<\omega t<90^{\circ}\right)$. In all smooth-wall tests, lower levels of turbulence coincide with the wall-flow reversal in the range $60^{\circ}<\omega t<90^{\circ}$, as detailed previously by Batchelor (1967) and several experimental studies (e.g. Carstensen et al., 2010; Jensen et al., 1989).

The main driver in the formation of vortex tubes, as discussed in Carstensen et al. (2010), is the Kelvin-Helmholtz instability which develops at the interface between two layers of the same fluid flow at dissimilar velocities (including in opposite directions). This scenario results in the formation of a peak in the vertical profile of the viscous shear stress. The location of this peak may indicate the position of the centre of the vortex tube if the Kelvin-Helmholtz instability is fulfilled. Figure 5 presents the streamwise total shear-stress vertical profile obtained for Test 2 (only half a cycle is plotted but it is representative of the entire period of oscillation, being symmetric for the rest of the cycle). Although Test 2 is within the laminar-to-turbulent

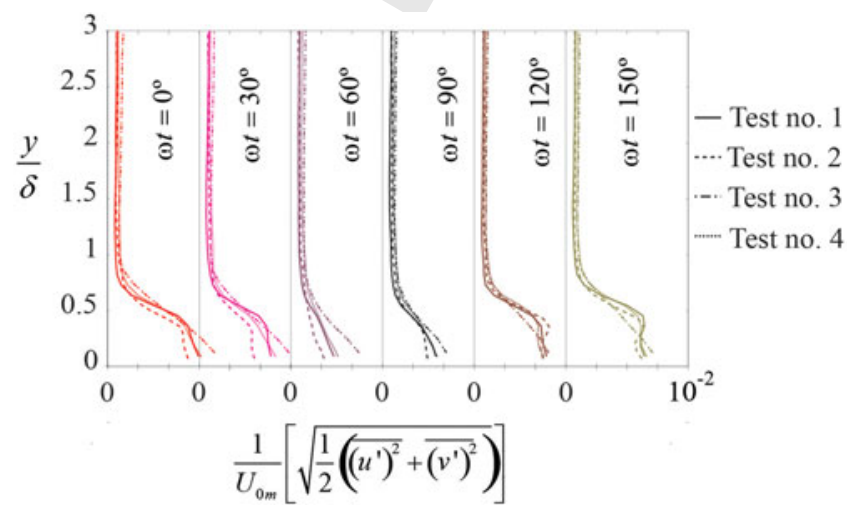

Figure 4 Vertical profiles of the relative turbulence intensities as a function of phase for the smooth-wall experiments. Square fluctuations are phase-averaged and also streamwise averaged

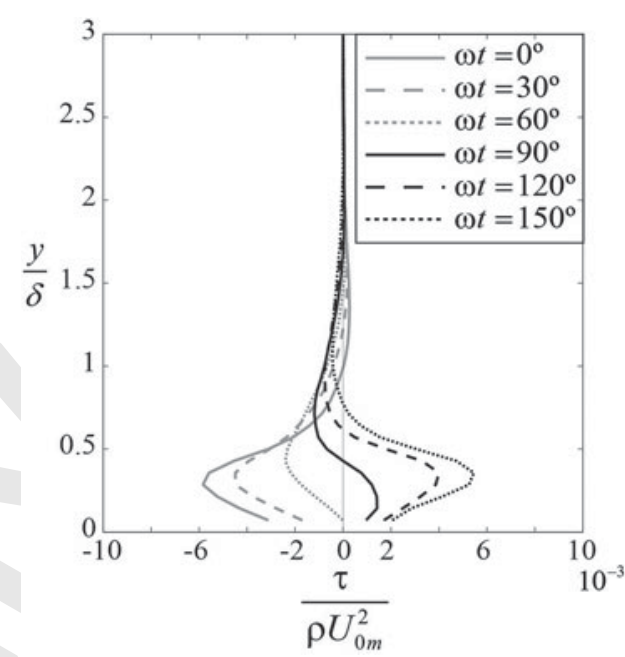

496

501

506

Figure 5 Vertical profiles of total shear stress-streamwise averaged, $\tau=\tau_{v}+\tau_{R^{-}}$profiles from Test 2 , smooth wall, $\mathrm{R}=1.27 \times 10^{4}$. Half cycle

regime, Mujal-Colilles et al. (2013) reported low levels of turbulence in the vicinity of the wall. Therefore, beyond the first point closer to the wall, the total shear stress is entirely due to viscous effects and the profiles in Fig. 5 can be fit with the second derivative of the Reynolds averaged Navier Stokes (RANS) streamwise velocity profile equation for laminar oscillatory flow over smooth beds:

$$
U_{0}(y, \omega t)=-U_{0 m}\left[\cos (\omega t)-e^{\delta_{1} y} \cos \left(\omega t-\delta_{1} y\right)\right]
$$

where $\delta_{1}=\sqrt{\nu T / \pi}$ is the Stokes length.

Using Eq. (4) after some mathematical manipulation, the conditions for the existence of the Kelvin-Helmholtz instability holds when $\mathrm{d}^{2} U_{0} / \mathrm{d} y^{2}=0$ and $\left(U-U_{0}\right) \mathrm{d}^{2} U / \mathrm{d} y^{2}<0$ (CushmanRoisin, Gualteri, \& Mihailovic, 2008). According to several previous studies (e.g. Carstensen et al., 2010; Jensen, 1988; Mujal-Colilles et al., 2013), the flow reversal close to the wall occurs between $\omega t=60^{\circ}$ and $\omega t=90^{\circ}$, coincident with the formation of a new peak seen in the total shear stress profile as indicated in Fig. 5. Pedocchi et al. (2011) described the pres- Q ence of shear layers emerging during the deceleration phases as inflection points in the phase-averaged velocity profiles. These shear layers become more intense when the flow reverses close to the wall and are linked to zero shear stress at the interface between the shear layers. At $\omega t=90^{\circ}$, the shear-stress profile presents a clear peak around $y / \delta \approx 0.2$ that propagates away from the wall during subsequent phases. This inflection point is still visible for $\omega t=0^{\circ}$ at $y / \delta \approx 0.4$ and continues migrating while sharpening, until $\omega t=60^{\circ}$, where the cycle begins again. However, the phase $\omega t=90^{\circ}$ shows the existence of a new peak as well as the prevalence of the maximum at $y / \delta \approx 0.75$ from the previous cycle with opposite sign, which slowly dissipates in magnitude while migrating upwards. This behaviour indicates that two types of vortex tubes may be simultaneously 
present in the flow: "new" structures created during the current flow reversal close to the wall, and "old" structures formed during flow reversal at an earlier phase migrating upwards and dissipating.

The snapshots of the phase-averaged swirling strength compared with the streamwise-averaged vertical shear stress profiles are helpful to better describe the formation and lifetime of the vortex tubes. In Fig. 6a, both peaks in shear stress correspond exactly to the centres of structures located at $y / \delta \approx 0.2$ and $y / \delta \approx 0.75$ noted in the phase-averaged swirling-strength field. Moreover, the height of zero shear stress location coincides with the division between these layers of swirling strength and indicates the division between the "old" and the "new" vortex tubes. In Fig. 6b, the negative peak in the shear-stress profiles is smoothed and the swirling strength snapshots show some residual turbulence but devoid of spatial coherence. Finally, in
Fig. 6c, the upper peak has almost disappeared from the shearstress profile and no regions of non-zero swirling strength are present beyond $y / \delta \approx 1.0$. These observations are entirely consistent with the existence of the Kelvin-Helmholtz instability leading to the formation of vortex tubes.

The presence of "old" and "new" vortex tubes at the same phase suggests that the life of these structures is longer than half a cycle. Figure 7 presents the correlation of swirling strength (background variable) and vorticity (colour contours) of the first half cycle (note that the entire cycle is actually represented because $\omega t>180^{\circ}$ is symmetric in the plots of Fig. 7a-f). Figure $7 \mathrm{a}$ shows the vortex tubes that were already created during the wall flow reversal of the previous half cycle, $\omega t<0^{\circ}$. New vortex tubes are created during the wall flow reversal in Fig. $7 \mathrm{c}$ and $\mathrm{d}$ at $y / \delta \approx 0.2$. These vortices grow in size while they remain close to the wall, but the centre of the tubes moves

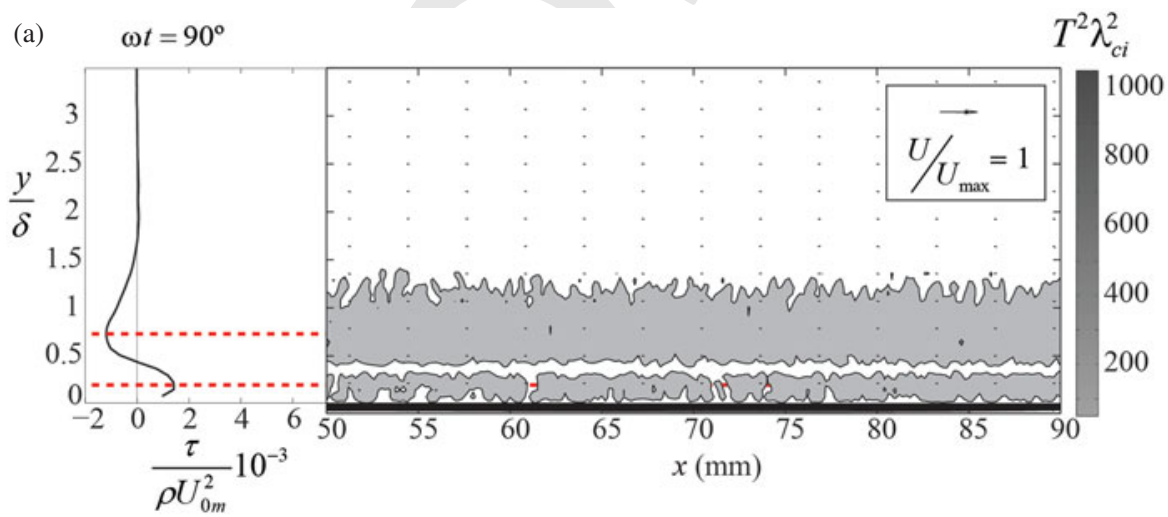

(b) $\quad \omega t=150^{\circ}$
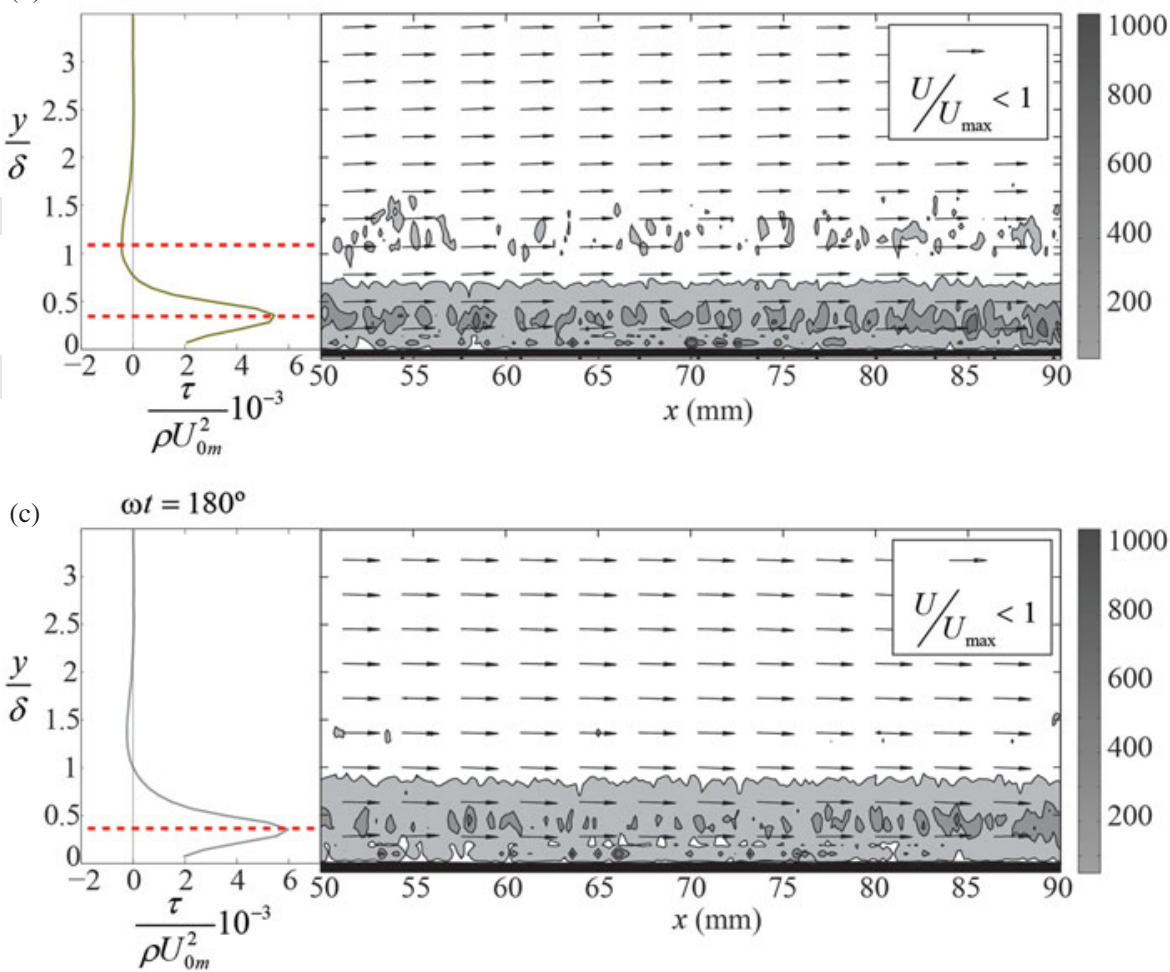

Figure 6 Combination of streamwise averaged shear stresses - left column, and phase-averaged velocity field with $\lambda_{c i}^{2} \cdot T^{2}$ as a background variable. Test 2 , smooth wall, $\mathrm{R}=1.27 \times 10^{4}$. Half cycle 


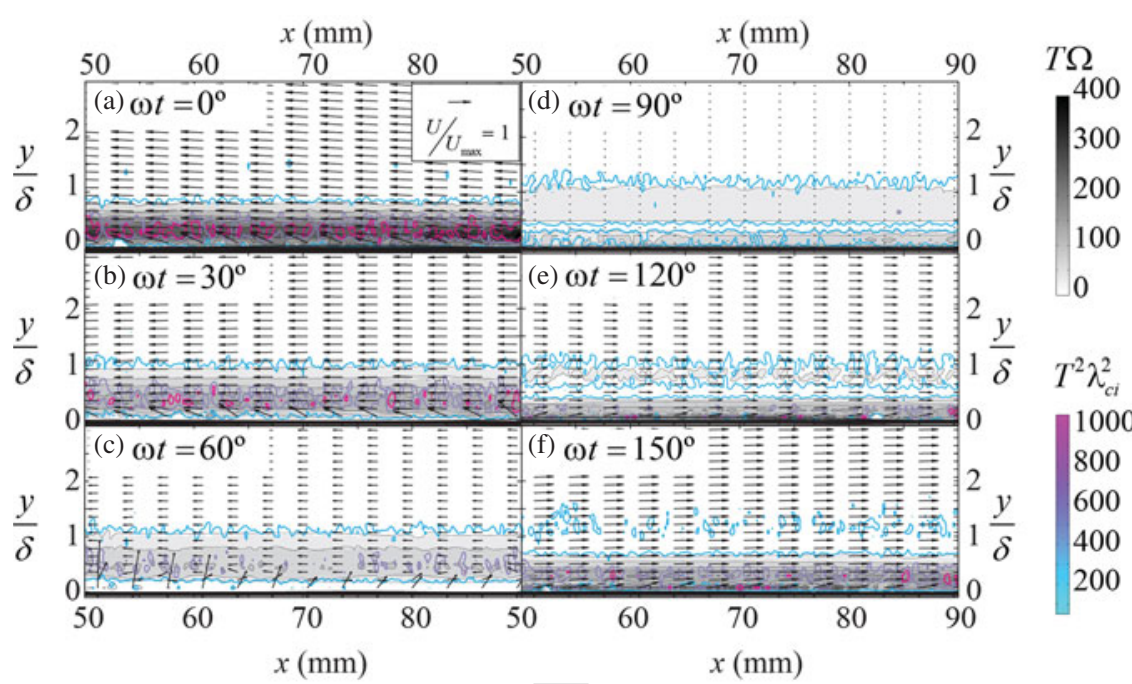

Figure 7 Phase evolution of the correlation between non-dimensional vorticity $(T \cdot|\Omega|)$ - background variable; and the non-dimensional swirling strength $\left(\lambda_{c i}^{2} \cdot T^{2}\right)$-contour variable). Test 2 , smooth wall $\mathrm{R}=1.27 \times 10^{4}$. Half cycle. The flow in (a), (b) and (c) is from right to left with decreasing velocity, (d) is the free stream flow reversal and in (e) and (f) the flow is from left to right

upward, as seen in Fig. 5. The detachment of the vortex tubes occurs when the new structures are formed close to the wall. Therefore, the life of the vortex tubes appears to be in the range $\omega t \approx 60^{\circ}$ to $\omega t<300^{\circ}$, representing three quarters of a cycle. Carstensen et al. (2010) reported that the vortex tubes they detected survived less than a quarter of a cycle, being dissipated around $\omega t \approx 120^{\circ}$ based on dye-based flow visualization. This observation may be biased by dilution of the dye owing to intense mixing and thus a lower estimate of vortex lifetime compared to that reported herein.

\subsection{Rough wall}

Few studies have considered the existence of structures in rough-wall oscillatory boundary layers. The first experimental work of Keiller and Sleath (1976) focused on regular rough surfaces using hemispheres. This was later reproduced numerically by Fornarelli and Vittori (2009) who also compared their results to another experimental work carried out using square roughness elements (Krstic \& Fernando, 2002). Sleath (1987) and Jensen et al. (1989) revealed the existence of jets of fluid emanating from irregular sediment beds, indicating the possibility of coherent structures created close to the bed and ejected outward during the oscillatory motion.

The recent experimental study of Carstensen, Sumer, and Fredsøe (2012) describes the existence of turbulent spots in the range $5 \times 10^{4}<\mathrm{R}<10^{5}$ motivated by their original work with smooth-wall oscillatory boundary layers (Carstensen et al., 2010). Turbulent spots were first described for unidirectional flow by Emmons (1951) as structures that are formed after strong fluctuations randomly positioned in space. Hladík, Jonáš, and Uruba (2011) presented PIV measurements that offered a detailed description of turbulent spots in both side and plan views. As with the present smooth-wall conditions, the present rough-wall conditions do not fall within the limits described by
Carstensen et al. (2012) for the formation of turbulent spots. Moreover, the lack of plan information in the current work does not allow us to confirm the existence of turbulent spots which have a well-defined character in plain view. Thus, though we identify spatial structures in the side-view PIV velocity fields that share some consistency with turbulent spots, we instead simply refer to them as turbulent bursts, which reflects the occurrence of spatially-coherent events associated with strong and intermittent turbulent behaviour in the side views presented herein.

The analysis performed to describe the coherent structures for rough-wall experiments follows the same procedures as the previous analysis for the smooth-wall experiments. The use of the phase-averaging may erase imprints of instantaneous turbulent spots, as described by Carstensen et al. (2010). On the other hand, the existence of regions with the consistent swirling strength can be explained by assuming that the same type of structure is being formed in the vicinity of the same location for all experiments, as will be detailed below.

Observations for the two different irregular rough beds showed three types of coherent structures:

(1) vortices: structures created during the wall flow reversal that are ejected as vortices and ultimately become bursts;

(2) turbulent bursts: structures that emerge like vortices during the flow reversal at the wall but are not strong enough to maintain coherence; these structures are ejected as bursts and reach lower heights compared to vortices $\theta$;

(3) shear layers: these features were only detected in experiments with $A /\left(2 D_{50}\right)<15$, and consist of several vortices shed continuously from particular grains at the wall; at the wall flow reversal stages, vortices 0 are formed at the same distance from the remaining structure of the shear layer. 
Vortices

In the present rough-wall experiments, vortices are formed during the flow reversal close to the wall, exhibiting similar characteristics as the vortex tubes described in section 0 for smooth-wall flow. However, several differences arise due to the presence of a rough bed.

The formation process of a representative vortex is plotted in Fig. 8 which presents the phase-averaged velocity field computed from 60 independent cycles of Test 6 (see supplementary material Test 6), with the small roughness and an amplituderoughness ratio $A / k_{s}=33$. Therefore, the detection of a vortex
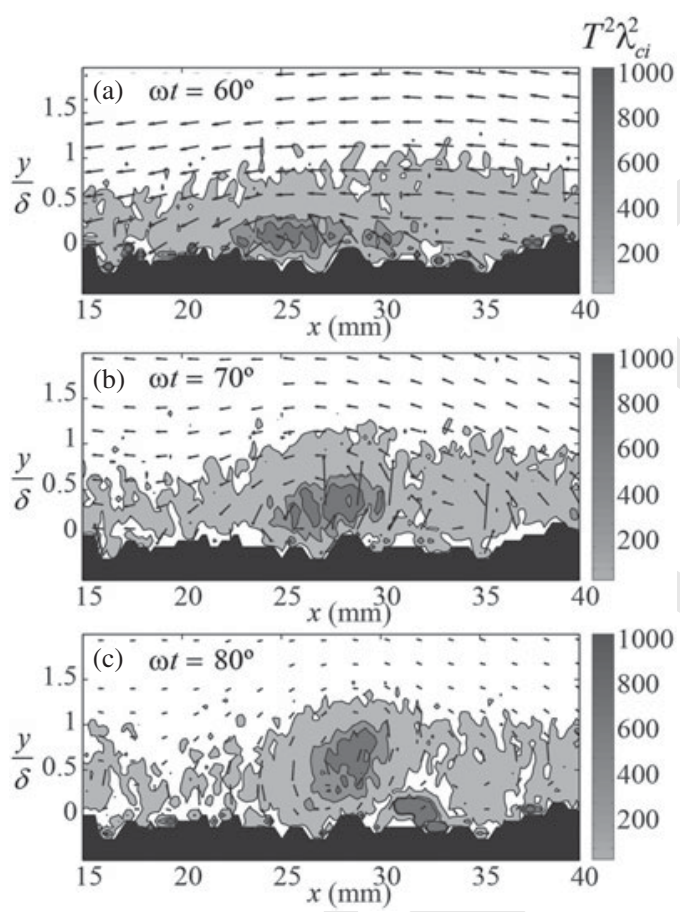

Figure 8 Formation of a vortex in Test 6; roughness: $D_{50}=1.5 \mathrm{~mm}$; $\mathrm{R}=1.27 \times 10^{4}$. Background variable contour-plots of dimensionless swirling strength $\lambda_{c i}^{2} \cdot T^{2}$ in the phase-averaged flow fields indicates the existence of this vortex, with similar spatial characteristics, in a large number of the instantaneous velocity fields at that phase. In the vicinity of a grain, flow separation occurs around $\omega t=70^{\circ}$ and forms a clear vortex. In contrast to vortex tubes formed in smooth-wall oscillatory flows, the phenomena associated with the origin of vortices in rough wall flows is clearly related to negative pressure gradients just downstream of some sediment grains, and therefore the location of vortex formation is directly correlated to specific grains of the bed. Locally, the detachment of the boundary layer at the top of the grains, seen in Fig. 8a, creates a wake transformed into a vortex due to the flow reversal close to the wall, which is clearly seen in Fig. $8 \mathrm{~b}$ and c. Once the flow close to the wall is fully reversed (Fig. 8c), the detachment of the boundary layer at the top of the same grain occurs in the opposite direction compared to Fig. 8a. In other studies, including Fornarelli and Vittori (2009), with regular rough walls, vortices were formed behind all hemispheres during the wall flow reversal. Herein, there exists a preferential formation of vortices from specific grain features owing to the irregularity of the bed roughness considered.

After being formed, these vortices detach from the wall and migrate upwards until a certain height with progression in phase. Figure 9 presents the evolution and dissipation of the same vortex shown in Fig. 8, where the vortex is highlighted with a red ellipse. The vortex sheds from the wall at $\omega t=90^{\circ}$ and loses some of its spatial coherence. Before dissipating around $\omega t=130^{\circ}$, the vortex travels as a simple burst along a welldefined upward path. Only two clear vortices are formed within this field of view, although one can identify other features in these fields that will be addressed later.

These results are consistent with the numerical observations of Fornarelli and Vittori (2009), particularly that vortices are formed behind specific sediment grains where pressure is minimum during the near-wall flow reversal, but pressure gradients are higher. Krstic and Fernando (2002) described a dipole-like

Figure 9 Time evolution of the position of a vortex, ellipse, and a turbulent burst, rectangle, created during the wall flow reversal $\omega t=70^{\circ}$. Background variable: $\lambda_{c i}^{2} \cdot T^{2}$. Test 6; roughness: $D_{50}=1.5 \mathrm{~mm} ; \mathrm{R}=1.27 \times 10^{4}$ 
vortex formed during the wall flow reversal which was not detected in any of the cases presented herein. This may be attributable to the shape of the cubic roughness elements used by Krstic and Fernando (2002) which may have led to the formation of a second vortex after the first vortex was formed. In fact, the shedding process of the dipole-like vortices described by Krstic and Fernando (2002) coincides with that shown in Fig. 9 in which vortices are elongated while propelling from the wall. Vortices were present in all the experiments, with vortices trajectories not differing from the ones described for Test 6 .

Figure 10 presents the phase evolution of the streamwise and wall-normal positions of the vortex centre, defined here as the centre of the zone with constant swirling strength $\left(T \lambda_{c i}^{2}>50\right)$. In general, larger roughness scenarios resulted in longer vortex burst lifetimes. Clear distinctions can be seen for the different bed scenarios, but among all tests there is similarity in profiles belonging to tests with the larger $A / k_{s}$ ratio (Test 5 and Test 6; Test 7 and Test 8). Focusing on the streamwise vortex displacement with phase, Fig. 10a, higher velocities at latter stages are found in tests with larger amplitude, regardless of the roughness size of the bed (Test5 and Test 6; Test 9 and Test 10; see also supplementary material Text 10 ). This is likely due to the fact that the vertical location of the vortices is further from the wall where the mean streamwise velocity is also higher. The evolution of the vertical vortex position (Fig. 10b) displays an inflection point close to the free-stream flow reversal stages $\left(\omega t=90^{\circ}\right)$, indicating that the detachment from the wall is complete by this phase. This is more notable for higher-amplitude experiments with a small roughness bed size (Test 5 and Test 6 ). The evolution of the vertical position combined with the horizontal displacement shows that higher horizontal velocities are reached once the detachment complete. The dependence of the vortex horizontal and vertical position evolution on the amplitude (Fig. 10b), as well as on the roughness size, shows that these two variables can be defined as characteristic lengths of the flow.

\section{Turbulent bursts}

Turbulent bursts are defined separately from vortices due to their different spatial characteristics in the phase-averaged velocity fields. Figure 9 displays the trajectory of a turbulent burst (green square), compared to the vortex trajectory in Test 6 with small roughness elements. It is important to highlight that Fig. 9, like Fig. 8, presents phase-averaged swirling strength fields. Thus, the representation of a turbulent burst in the phase-averaged flow field is likely the result of several vortices forming near the same position but being offset in different cycles. However, when we look at each experiment, the zone where a turbulent burst is located does not show a clear vortex, indicating that it is indeed a burst.

The first difference between turbulent bursts and vortices described in the previous section is the coherence of the vortex itself. That is, bursts may be created as vortices but the coherence of the vortex is lost even before the structure detaches from the wall. Also, some of the turbulent bursts are not born as a clear vortex but rather simply as a spatially-coherent region of high turbulence levels. The trajectory of turbulent bursts is similar to that of vortices. They are created close to the wall during the near-wall flow reversal, and shed from the wall rapidly. However, turbulent bursts reach higher elevations than vortices once they have been shed from the wall, as seen in Fig. 9. The snapshot presented in Fig. 9d shows the existence of three vortices moving at a central height of about $y / \delta \approx 1.0$ and two bursts at a height $y / \delta \approx 0.5$.

The location of turbulent bursts relative to vortices is thought to be random. Other experiments do not show this pattern which may be related both to the shape of the gravel particles and the specific characteristics of the flow regime. It is important to recall that experiments performed with the small roughness sediment bed have been characterized as flows in the laminarto-turbulent transition, and that flows with the larger roughness are closer to the rough-wall flow regime. Therefore, none of the flows are fully rough when turbulent bursts and vortices are created during the wall flow reversal, and so the bed roughness has a significant influence on the location and evolution of the structures formed near the wall.

Figures 11 and 12 show the same snapshots as Fig. 9 detailing the position of the vortices and the turbulent bursts along a quarter of a cycle where both are formed, shed from the wall and move with the flow. From Fig. 11, where the distribution of the total shear stress is shown, another difference between vortices and turbulent bursts can be identified, whereby peaks of shear stresses occur near the centre of a vortex, particularly at the early stages of inception. On the other hand, no
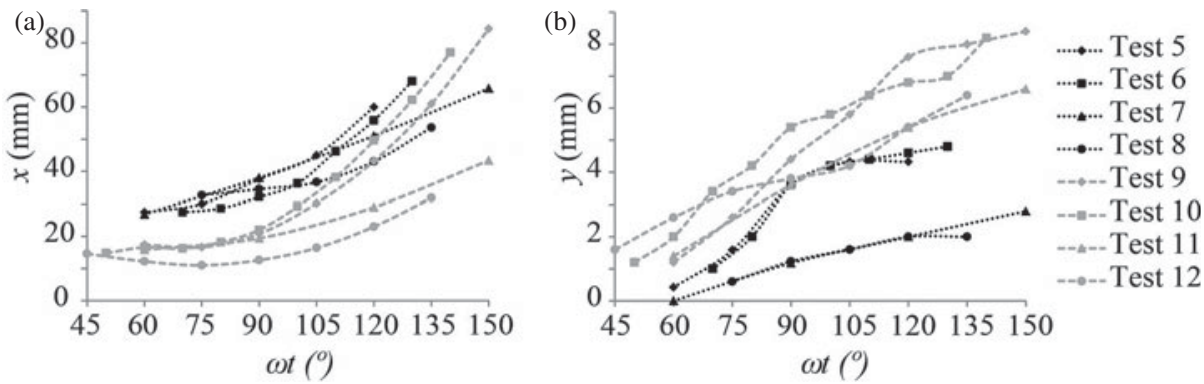

Figure 10 Phase evolution of the centre of one vortex in all experiments. (a) Horizontal position; (b) vertical position. Black lines: small roughness; grey lines: large roughness 


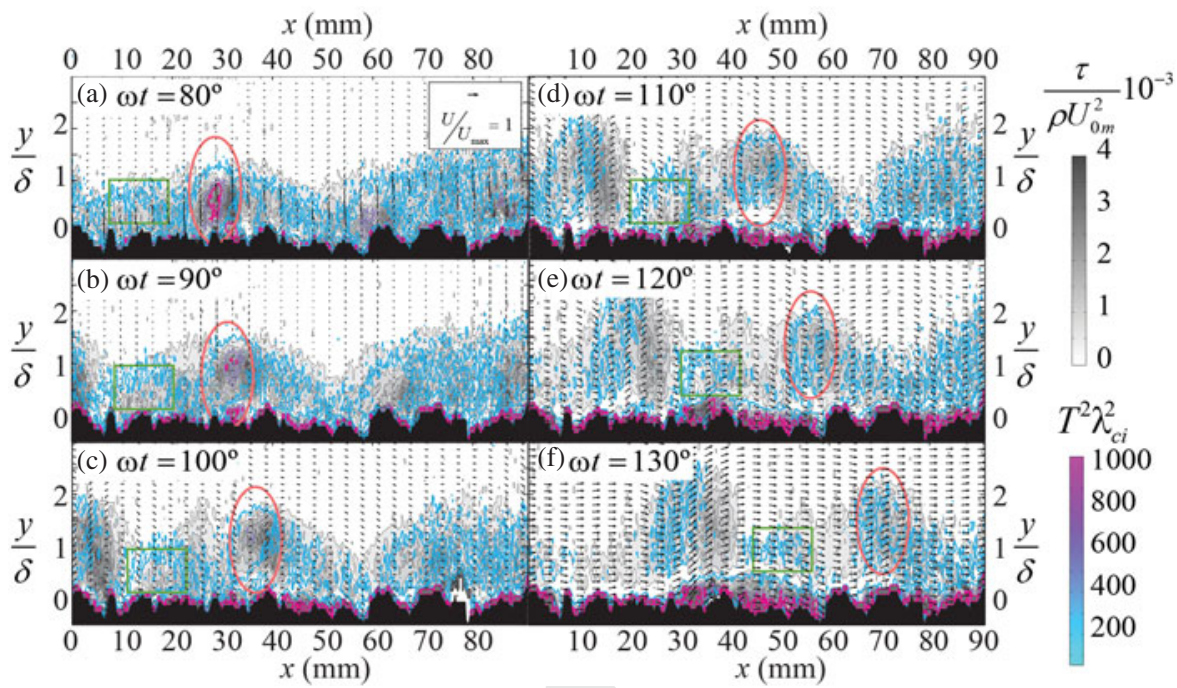

Figure 11 Phase evolution of the position of a vortex (ellipse), and a turbulent burst (rectangle). Background variable: $\left(\tau_{v}+\tau_{R}\right) / \rho U_{0 m}^{2} ;$ contour variable: $\lambda_{c i}^{2} \cdot T^{2}$. Test $6 ; D_{50}=1.5 \mathrm{~mm} ; \mathrm{R}=1.27 \times 10^{4}$

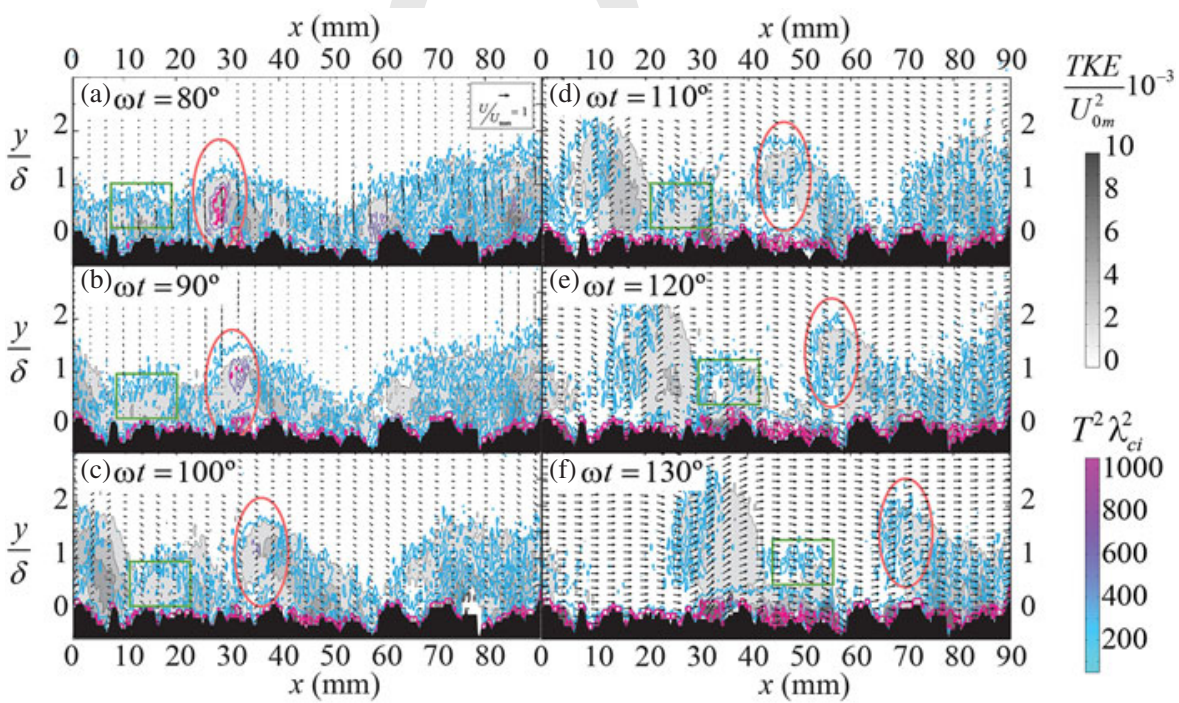

Figure 12 Phase evolution of the position of a vortex (ellipse), and a turbulent burst (rectangle). Background variable: $T K E / U_{0 m}^{2}$. Contour variable: $\lambda_{c i}^{2} \cdot T^{2}$. Test $6 ; D_{50}=1.5 \mathrm{~mm} ; \mathrm{R}=1.27 \times 10^{4}$

large gradients of velocity are detected coincident with the locations of turbulent bursts. In addition, higher values of shear stress vanish after $\omega t=110^{\circ}$ which coincides with the phase when vortices lose their spatial coherence and begin behaving as bursts (Fig. 9). In Fig. 12, turbulence kinetic energy (TKE) is plotted as the background variable showing how vortices concentrate maximum values of TKE on their upper-right sides in the direction of the flow at each phase. Likewise, turbulent bursts have higher values of TKE at their leading edge. However, the highest values of TKE are found in locations coincident with vortices, showing that these features concentrate larger amount of turbulence which, in turn, contributes to their longer lifetimes. This last observation represents another fundamental difference between turbulent bursts and vortices: the levels of turbulence are higher in vortex structures and persist longer than the same for turbulent bursts.
Carstensen et al. (2012) described turbulent spots as $3 \mathrm{D}$ structures randomly located in the plan view that form close to the wall during the wall flow reversal. Carstensen et al. (2010) reported that turbulent spots first occur during the bed shear stress reversal, grow in size and eventually merge together. Spikes in the shear stress signal were detected when the turbulent spot passed over a hot-film probe in their experiments. Mazzuoli et al. (2011) performed 3D numerical simulations and described the turbulent spots in oscillatory flow over smooth beds in the vertical plane. Based on previous descriptions, the structures detected in this research have been found to present similar characteristics in the side-view plane as the ones described by Mazzuoli et al. (2011) for smooth-wall conditions, mainly in the evolution of the structure once it has detached from the wall. However, as seen in Fig. 11, higher values of the shear stress are found to coincide with vortices rather than 
1101 turbulent bursts. Bearing in mind that the present analysis is based on phase averaging of 60 cycles, at an instantaneous cycle the shear stress could be as high as the one for the vortex coherent structure, but spatial intermittency coupled with the phase averaging may somewhat suppress this result.

\section{Shear layers}

Shear-layer signatures are only present in the experiments with larger roughness elements, $D_{50}=7.0 \mathrm{~mm}$ (Tests 9, 10, 11 and 12) and are found to be related to vortices and turbulent bursts. However, in the vicinity of the grains, the flow is stronger at smaller roughness for vortices to be shed and therefore, the flow conditions for larger roughness experiments are not strong enough to cause flow separation from all the grains in the same magnitude as it does for smaller roughness experiments.

Shear layers consist of a sequence of vortices shed from specific grains of the rough wall. This is observed in Fig. 13a where a shear layer is formed at the location $x \approx 25 \mathrm{~mm}$. This signature is linked to vortices described previously as a single coherent structure: the shear layer forms after the generation of multiple single vortices shedding from the vicinity of a particular grain during the acceleration phases. Thereafter $(\omega t>0)$, the angle of the shear layer increases until the onset of the wall flow reversal (Fig. 13a, b and c). At this phase, the vortex is formed with the remaining turbulence from the shear layer and shed from the wall during latter phases (Fig. 13d, e and f). Simultaneously, another shear layer forms from a different grain with an opposite directional sense and grows continuously until the maximum free-stream velocity phase. During the acceleration process, the shear layer grows in size instead of increasing in angle as no significant angle can be identified at the early stages of initiation.

It is not entirely clear if the shear layer shown in Fig. 13a Q3 is originated from a single grain. In a closer view of Fig. 13a shear layers, previously described, seem to originate from more than one grain of sediment. In fact, when observing previous stages in detail in Fig. 15, the shear layer is formed from one grain at early stages of the acceleration quarter cycle, when the flow is laminar. Then, as the free-stream flow and near wall velocity increase, the shear-layer covers the grain reaching the next grain similar to that in Fig. 13e. Thereafter, once

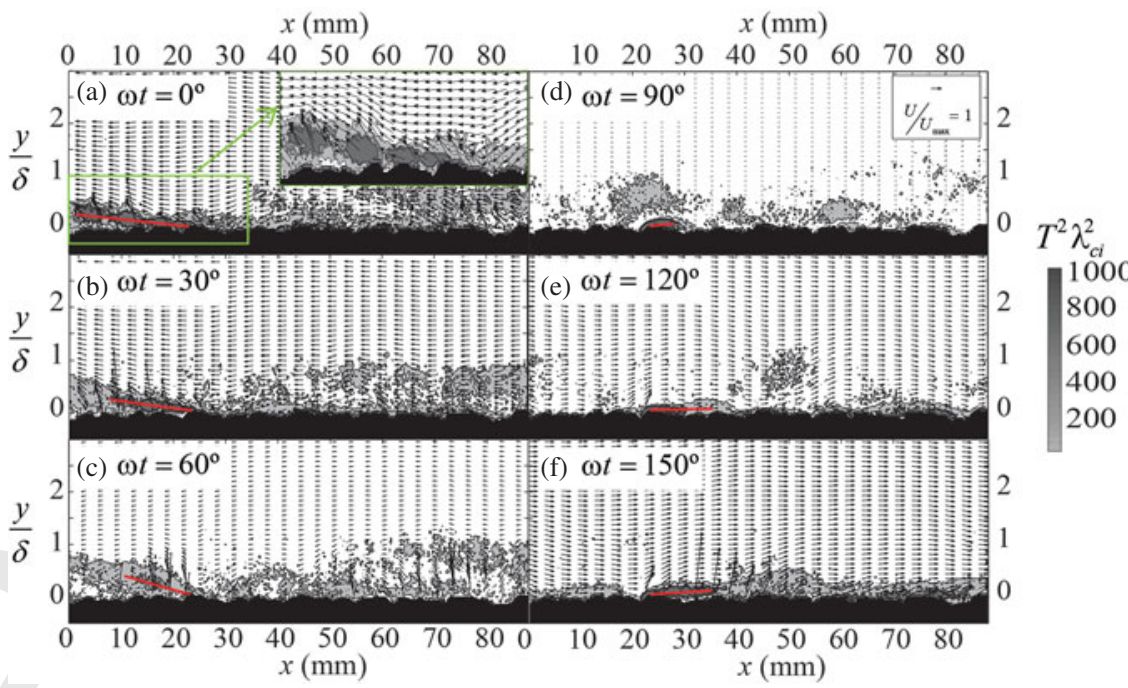

$x(\mathrm{~mm})$

$x(\mathrm{~mm})$

Figure 13 Phase evolution of a shear layer formed in Test 10 ; roughness: $D_{50}=7.0 \mathrm{~mm} ; \mathrm{R}=1.27 \times 10^{4}$. Half cycle. Background variable: $\lambda_{c i}^{2} \cdot T^{2}$

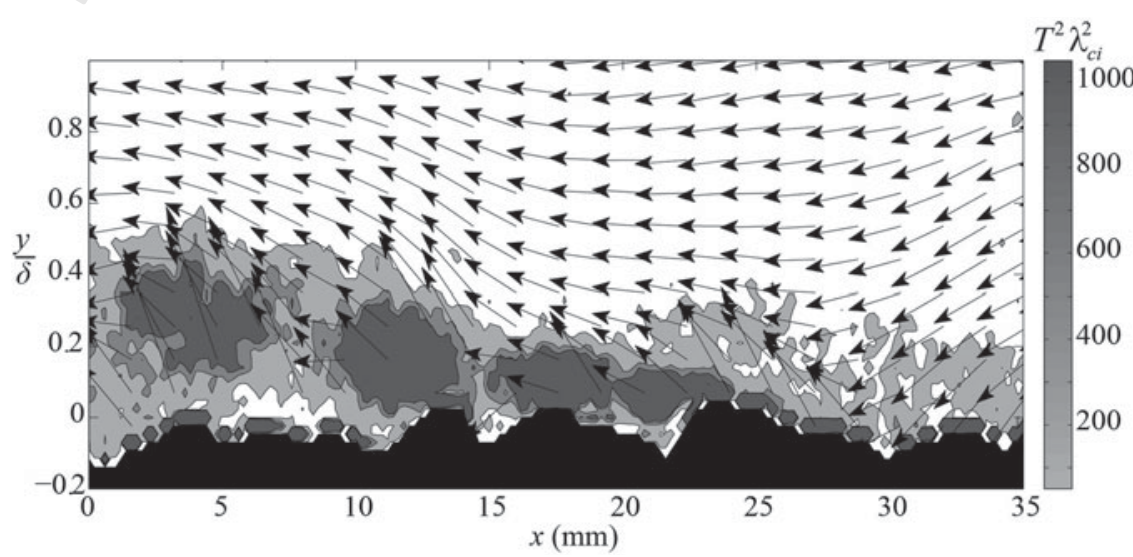

Figure 14 Close view of Fig. 13a. Shear layer at $\omega t=0^{\circ}$. Background variable: $\lambda_{c i}^{2} \cdot T^{2}$. Test $10 ; D_{50}=7.0 \mathrm{~mm} ; \mathrm{R}=1.27 \times 10^{4}$ 


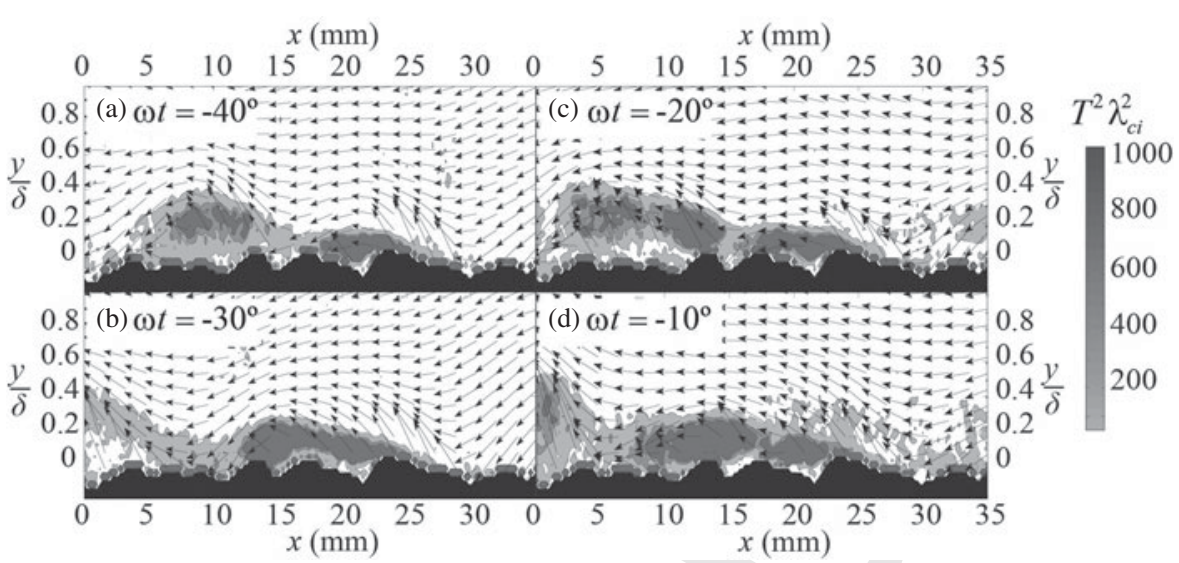

Figure 15 Close view at stages $\omega t<0^{\circ}$ during the acceleration process. Background variable: $\lambda_{c i}^{2} \cdot T^{2}$. Test $10 ; D_{50}=7.0 \mathrm{~mm} ; \mathrm{R}=1.27 \times 10^{4}$

the local velocity has increased, the shear layer detaches from the wall at the second grain (Fig. 15a) as a vortex. In Fig. 15b, the boundary layer formed at the original grain is detached but does not have enough energy to shed from the wall until it reaches a third grain in Fig. 15c. Finally, when the free-stream flow is at maximum, the shear layer becomes stable without any waviness and is comprised of several vortices from different grains, although the boundary layer detaches originally from one grain. Other tests showed similar behaviour on the initiation and development of the shear layer. Moreover, higher Reynolds number cases showed a complete development of the shear layer at previous phases. Finally, the fact that vortices are shed from certain grains at pre-flow reversal and other grains at post-flow reversal implies that the specific shape of the grains upstream and downstream of the local oncoming flow plays a defining role in determining whether local flow separation will occur.

\section{Conclusions}

The PIV experiments detailed herein facilitated the study of the formation and evolution of coherent structures present in oscillatory flows over smooth and rough walls. In contrast to previous qualitative flow-visualization studies, PIV allowed quantification of the characteristics of the underlying coherent motions for a range of wall conditions.

Features present in oscillatory flows over a smooth wall were confirmed to be vortex tubes, defined within the limitations of 2D planar PIV. Similar to the experimental work of Carstensen et al. (2010), features detected in the experiments within the laminar to turbulent transition regime were formed by the Kelvin-Helmholtz instability during the wall flow reversal. As outlined by several authors (i.e. Jensen et al., 1989; MujalColilles et al., 2013; Sarpkaya, 1993), the flow reversal close to the wall occurs before the free-stream flow reversal. Therefore, there exists a gradient in the vertical velocity profiles, reflected in the shear stress profiles, in which very low values of turbulent stresses were detected to trigger the Kelvin-Helmholtz instability. During the second half cycle, where another near-wall flow reversal occurs, vortices present from the previous half cycle merge and migrate upwards while new structures form near the wall.

Three different coherent structures were identified in phaseaveraged representations of the rough-wall flows: vortices, turbulent bursts, and shear layers. Vortices appeared during the wall flow reversal and were shed from the wall. Beyond this point, their coherence as a vortex disappeared and transitioned into a burst. Thus, these bursts originated as clear vortices close to the wall and maintained a certain height, particularly in the smaller roughness scenarios. The trajectory of vortices was found to depend, ultimately, on the roughness diameter $k_{s}$ and the amplitude of oscillation, $A$, although the formationevolution-decease process was common in all the experiments. Thus, vortices were created during the latter stages of the deceleration phases and ejected from the wall during early acceleration stages. Vortices disappeared before the next decelerating cycle began, lasting, at most, a quarter of a cycle including the stages in which they lost their vortex appearance and became bursts.

The second feature defined was linked to vortices, but was termed bursts. Bursts consisted of smaller vortices created close together in different instantaneous fields that appeared as a spatially-coherent region of high turbulence in the phaseaveraged snapshots. Bursts also originated at the wall but did not extend into the flow as far as the vortices did prior to dissipating. However, their life cycle was equivalent to that of the shed vortices.

Finally, the third coherent structure observed was a shear layer that formed during the maximum free stream velocity stages. Shear layers were only detected in experiments with $A / k_{s}<15$ and were linked to vortices described as the first structure. Thus, shear layers formed just as vortices shed from some selected grains of the irregular rough bed. Initially they were considered laminar structures covering a single grain. However, as the streamwise velocity increased, this attached 
1321 structure eventually detached from the grains. At this phase, small vortices were formed, and the shear layer became a chain of vortices formed at a specific grain, colliding into the next crest and subsequently detaching. This was clearer at maximum free stream velocity phases. During decelerating stages, small vor1326 tices did not rebound on the following crests and the shear layer instead became a large vortex during the wall flow reversal. This behaviour was previously observed in experiments conducted in the same oscillatory flow facility but in the presence of ripples (Admiraal et al., 2004). Vortices developing in the lee side of 1331 the ripples and later advected by the oscillatory flow were found to play a major role in the mechanisms leading to the formation and evolution of finite amplitude bedforms.

In all experiments, phase averaging was used to identify structures in the mean (i.e. phase-averaged) flow which were 1336 also present in most of the instantaneous velocity fields. For smooth walls, this showed that vortices appear located at the same place. In rough walls, although the roughness was designed to be spatially uniform in its characteristics, structures were commonly shed from the same topographical features.

1341 This is likely due to the transitional flow regime studied herein (laminar to turbulent) in which particular roughness features can become important. Discrete shedding locations may also have resulted from structures forming during phases of very low free stream velocity. Recalling that the definition of the flow regime (laminar/turbulent) in oscillatory flows assumes that most of the phases are within one regime, some may be in laminar regime (particularly the flow reversal phases), during which features are formed and shed. It is in this situation where anomalies in the roughness appear to trigger signatures at the 1351 same position. It is important to highlight that structures randomly distributed in space are not analysed in this work due to the phase-averaging analysis that can mask most of them. However, some of the features defined in this research can also be the result of the phase averaging of small vortices located close one to the other but not at exact same location in each instantaneous field (i.e. turbulent bursts).

\section{Acknowledgements}

The experimental work presented herein was conducted during a one-year-long visit of Anna Mujal-Colilles to the Ven Te Chow Hydrosystems Laboratory at UIUC. The authors are also grateful to Julio Barros for sharing his image analysis code and to Jose M. Mier for his help during the experimental campaign.

\section{Funding}

13Ф\$ The financial support of the Ministerio de Educacion y Ciencia de España [grant CGL 2009-13039] is gratefully acknowledged. The support of the United States Office of Naval research [grant ONR N00014-11-1-0293] is also acknowledged.

\section{Supplemental data}

Supplemental data for this article can be accessed 10.1080/0022 1686.2016.1174960.

\section{Notation}

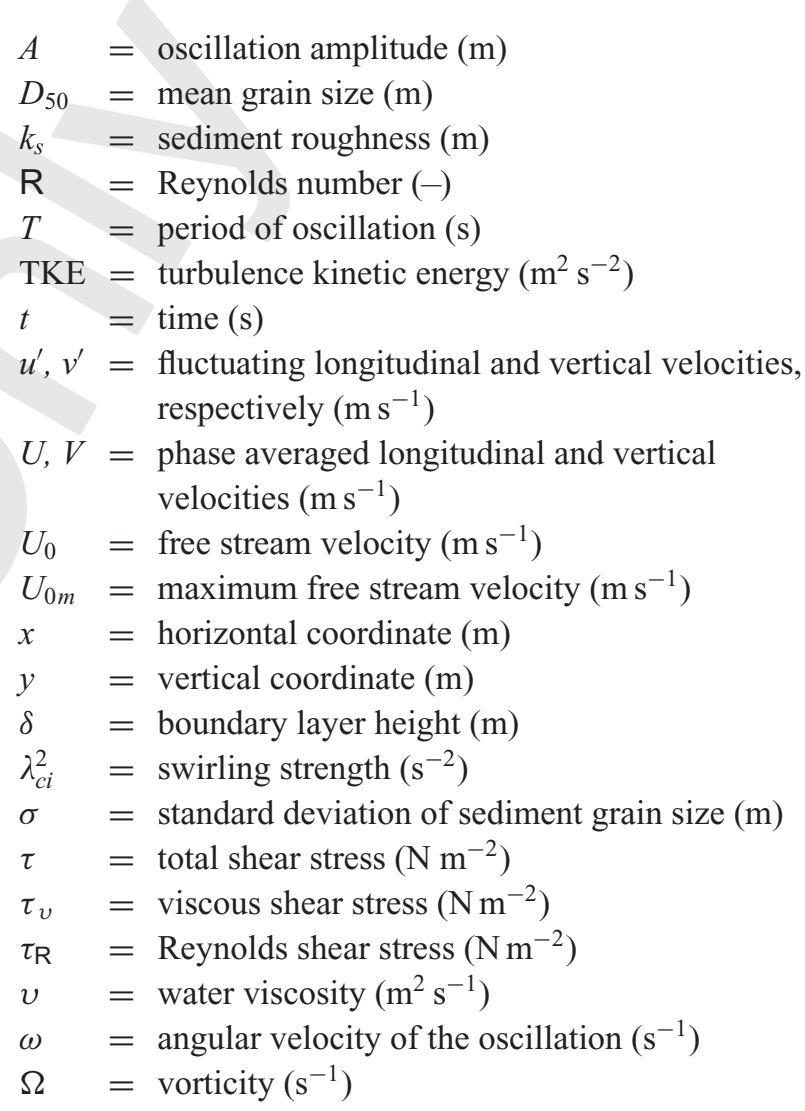

\section{References}

Admiraal, D. M., García, M. H., \& Musalem, R. (2004). Exploratory study of oscillatory flow over a movable sediment bed with Particle-Image-Velocimetry (PIV). In BridgesASCE (p. 93).

Adrian, R. J., Christensen, K. T., \& Liu, Z. (2000). Analysis and interpretation of instantaneous turbulent velocity fields. Experiments in Fluids, 29(95).

Batchelor, G. K. (1967). An introduction to fluid dynamics. Cambridge Univ $\mathrm{Pr}$.

Blondeaux, P., Scandura, P., \& Vittori, G. (2004). Coherent structures in an oscillatory separated flow: Numerical experiments. Journal of Fluid Mechanics, 518, 215-229.

Carstensen, S., Sumer, B. M., \& Fredsøe, J. (2010). Coherent structures in wave boundary layers. Part 1. Oscillatory motion. Journal of Fluid Mechanics, 646, 169.

Carstensen, S., Sumer, B. M., \& Fredsøe, J. (2012). A note on turbulent spots over a rough bed in wave boundary layers. Physics of Fluids, 24(11), 115104. doi:10.1063/1.4767536 
Costamagna, P., Vittori, G., \& Blondeaux, P. (2003). Coherent structures in oscillatory boundary layers. Journal of Fluid Mechanics, 474, 1-33.

Cushman-Roisin, B., Gualteri, C., \& Mihailovic, D. T. (2008). Environmental fluid mechanics. Leiden: Taylor \& Francis.

Emmons, H. W. (1951). The laminar-turbulent transition in boundary layers. Journal of the Aeronautical Sciences (Institute of the Aeronautical Sciences), 18, 490-498.

Fornarelli, F., \& Vittori, G. (2009). Oscillatory boundary layer close to a rough wall. European Journal of Mechanics B/Fluids, 28(2), 283-295.

Hino, M., Nakayama, A., Kashiwayanagi, M., \& Hara, T. (1983). Experiments on the turbulence statistics and the structure of a reciprocating oscillatory flow. Journal of Fluid Mechanics, 131, 363-400.

Hladík, O., Jonáš, P., \& Uruba, V. (2011). Dynamics of turbulent spots in transitional boundary layer. 13th European Turbulence Conference, September 2011, Poland. In Journal of Physics: Conference Series (Vol. 318, No. 3, p. 032028).

Jensen, B. (1988). Experimental investigation of Turbulent Oscillatory Boundary Layers. Insitute of Hydrodynamics and Hydraulic Engineering. Technical University of Denmark.

Jensen, B. J., Sumer, B. M., \& Fredsoe, J. (1989). Turbulent oscillatory boundary layers at high Reynolds numbers. Journal of Fluid Mechanics, 206(1), 265-297.

Jonsson, I. G. (1980). A new approach to oscillatory rough turbulent boundary layers. Ocean Engineering, 7(1), $109-152$.

Kamphuis, J. W. (1975). Friction factor under oscillatory waves. Journal of the Waterways Harbors and Coastal Engineering Division, 101(2), 135-144.

Keiller, D. C., \& Sleath, J. F. A. (1976). Velocity measurements close to a rough plate oscillating in its own plane. Journal of Fluid Mechanics, 73(04), 673-691.
Krstic, R. V., \& Fernando, H. J. (2002). The nature of rough-wall oscillatory boundary layers. Journal of Hydraulic Research, 39(6), 655-666.

Lamb, H. (1932). Hydrodynamics. Cambridge, UK: Cambridge University Press. Retrieved.

Li, H. (1954). Stability of oscillatory laminar flow along a wall. Beach Erosion Board. Corps of Engineers.

Mazzuoli, M., Vittori, G., \& Blondeaux, P. (2011). Turbulent spots in oscillatory boundary layers. Journal of Fluid Mechanics, 685, 365-376.

Mujal-Colilles, A., Mier, J. M., Christensen, K. T., Bateman, A., \& Garcia, M. H. (2013). PIV experiments in rough-wall, laminar-to-turbulent, oscillatory boundary-layer flows. Experiments in Fluids, 55(1), 1633.

Pedocchi, F., \& Garcia, M. H. (2009). Friction coefficient for oscillatory flow: The rough-smooth turbulent transition. Journal of Hydraulic Research, 47(4), 438-444.

Sarpkaya, T. (1993). Coherent structures in oscillatory boundary layers. Journal of Fluid Mechanics, 253, 105-140.

Sleath, J. F. (1987). Turbulent oscillatory flow over rough beds. Journal of Fluid Mechanics, 182, 369-409.

Sumer, B. M., Jensen, B., \& Fredsoe, J. (1987). Turbulence in oscillatory boundary layers. Advances in Turbulence (e. Gt. Comte-Bellot \& J. Mathieu), 556-567.

Vittori, G., \& Verzicco, R. (1998). Direct simulation of transition in an oscillatory boundary layer. Journal of Fluid Mechanics, 371(September 2000), 207-232.

Wu, Y., \& Christensen, K. T. (2006). Population trends of spanwise vortices in wall turbulence. Journal of Fluid Mechanics, 568, 55-76, 2006.

Zhou, J., Adrian, R. J., Balachandar, S., \& Kendall, T. M. (1999). Mechanisms for generating coherent packets of hairpin vortices in channel flow. Journal of Fluid Mechanics, 387(1), 353-396. 